\title{
Per3 VNTR polymorphism and chronic heart failure
}

\author{
Jolana Lipkova ${ }^{a}$, Julie Anna Bienertova-Vasku ${ }^{a}$, Lenka Spinarova ${ }^{b}$, Petr Bienert ${ }^{a}$, Marian Hlavna ${ }^{a}$, \\ Monika Pavkova Goldbergova ${ }^{a}$, Jiri Parenica', Jindrich Spinarc, Anna Vasku ${ }^{a}$
}

\begin{abstract}
Aims. The aim of this study was to investigate the relationship between gene Period3 (Per3) variable number tandem repeat (VNTR) polymorphism and chronic heart failure (CHF).

Methods. The study subjects (372 patients of Caucasian origin with CHF and 332 healthy controls) were genotyped for Per3 VNTR polymorphism using an allele-specific PCR.

Results. No significant differences in genotype or Per3 VNTR allele frequencies were found between CHF cases and controls $\left(P_{\mathrm{g}}=0.30, P_{\mathrm{a}}=0.52\right)$. No significant differences were uncovered either between $\mathrm{CHF}$ cases according to etiology (DCMP vs. IHD; $\left.P_{\mathrm{g}}=0.87, P_{\mathrm{a}}=0.91\right)$. In the multivariate regression modeling, no predictive function of VNTR Per3 polymorphism on ejection fraction or NYHA class, hyperlipidaemia or type Il diabetes risk was found.

Conclusion. Per3 VNTR polymorphism is not a major risk factor for chronic heart failure or a factor modulating the severity of the $\mathrm{CHF}$ in this population.
\end{abstract}

Key words: circadian clock, Per3, polymorphism, case-control study, chronic heart failure

Received: November 4, 2011; Accepted with revision: July 4, 2012; Available online: October 30, 2012 http://dx.doi.org/10.5507/bp.2012.069

a Institute of Pathological Physiology, Faculty of Medicine, Masaryk University Brno, Czech Republic

${ }^{b} 7^{\text {st }}$ Department of Internal Medicine - Cardioangiology, Faculty of Medicine and St. Anne's University Hospital, Brno

${ }^{c} 7^{\text {st }}$ Department of Cardiology, Faculty Hospital Brno

Corresponding author: Monika Pavkova Goldbergova, e-mail: goldberg@med.muni.cz

\section{INTRODUCTION}

Many physiological processes in the organism exhibit daily rhythmicity, which is generated by an internal "clock" setting about 24-h periodic expression of clock genes and their products. Such reliable biological oscillators allow almost every cell to predict environmental changes and thus accurately react to them. In humans, autonomic processes such as the control of sleep, body temperature, and hormone secretion, renal and cardiovascular functions are subject to circadian variation ${ }^{1-5}$.

Cell "clocks" are governed by a primary clock system in the suprachiasmatic nuclei (SCN), which reacts to daylight changes. At a molecular level this endogenous system consists of a series of positive and negative transcriptional feedback loops interacting with many clockdependent proteins and thus affecting different metabolic pathways ${ }^{6,7}$. According to the basic model of the function of "core clock proteins", the principal components of the positive loop BMAL1 and CLOCK periodically activate the transcription of Per and Cry genes. Therefore, the Per and Cry complexes, after reaching threshold concentration, interact with BMAL1 and CLOCK and repress their transcription therefore generating nearly antiphasic expression $^{8,9}$.

Despite the lack of precise knowledge of signaling pathways of all components integrated in the clock machinery, it has been experimentally proven in mammals that mutations in the "core clock genes" may lead to changes in circadian rhythmicity, disturbance of sleepwake cycles and may also affect heart control and blood pressure ${ }^{10,11}$. Period 3 (Per3), one of the key components of the negative limb of the human clock system, is a member of the Period protein family, described first in Drosophila ${ }^{12}$, and has recently been associated with metabolic dysfunctions. According to functional studies, deletion of Per3 in mice has a mild influence on circadian activity ${ }^{13}$. However, in humans, several mutations in Per3 have been described to affect sleep homeostasis and mood disorders ${ }^{14-17}$. Among the most surveyed Per3 polymorphisms in the last five years is the biallelic length change near the putative phosphorylation site of exon 18, composed of 4 or 5 indirect repeats. Numerous studies have linked this VNTR polymorphism to overall control of autonomic balance during sleep and wake cycles and indirectly to cardiac autonomic control ${ }^{18-20}$.

However, little is known about the exact clock mechanism in cardiovascular biology, and circadian rhythms in the heart have not yet been characterized at the transcriptional level, although several studies have shown that clock genes have an important function in myocardial contractile function and metabolism. Experiments in this area conducted to date have mostly involved manipulations of the light-dark cycle of mice clock mutants and their influence on myocardial functions ${ }^{21,22}$. Possible imbalance or impairment in the clock mechanism within the cardiomyocyte may alter the cardiac metabolism and function and thus increase the susceptibility of cardiovascular diseases. Therefore, we chose the Per 3 gene and decided to investigate the differences in allelic representation of VNTR polymorphism among subject with chronic heart failure $(\mathrm{CHF})$. 


\section{MATERIAL AND METHODS}

\section{Subjects}

The study cohort consisted of 372 consecutive patients (median of age $56 \mathrm{y}$, age range 21 - $91 \mathrm{y}, 107$ females/ 265 males) inclusive of chronic heart failure diagnosis (functional class NYHA II-IV, ejection fraction median $25 \%$, cardiothoracic index more than $50 \%$ ) both of ischemic heart disease (IHD) or dilated cardiomyopathy (DCMP) origin. To estimate the population frequencies of the examined genotypes and alleles, the control cohort was recruited consisting of 332 healthy individuals of similar age and gender distribution, without clinical signs of cardiovascular diseases and without a family history of early cardiovascular disease (median of age 51, age range 15.8 - 86, 218 females/ 189 males). The prevalence of diabetes mellitus and hyperlipidaemia in the controls was approximately $6 \%$ and $8 \%$, respectively, which corresponds well with the general prevalence in the Czech population. In all subjects, a complete medical history with respect to conventional cardiovascular risk factors was obtained. All patients had chronic heart failure of at least of 3 months in duration and were stable on unchanged medication for at least 1 month. All patients originated from the Czech Caucasian population and were recruited at the $1^{\text {st }}$ Cardioangiological and $2^{\text {nd }}$ Internal Departments, St. Ann's Hospital, Brno. The study was approved by the Committee for Ethics of Medical Experiments on Human Subjects, Faculty of Medicine, Masaryk University, Brno (no. 64/93, 1993) and was performed in adherence to the Declaration of Helsinki Guidelines. Participants gave their written informed consent before they entered the study.

\section{Clinical examination}

Clinical examination, basic and special laboratory, including renal functions, was done early in the morning followed by echocardiographic examination (SONOS 5500, Hewlett-Packard). The echocardiographic measurements were performed using a $2.5 \mathrm{MHz}$ phased array transducer, volumes were measured from apical four-chamber view and ejection fraction was calculated using the single plain Chapman method. Electrocardiography and standard X-ray (with evaluation of cardio-thoracic index and pulmonary congestion by the method of Meszaros) were obtained. Hyperlipidaemia was defined as plasma total cholesterol level of $>200 \mathrm{mg} / \mathrm{dL}$, plasma triglyceride level of $>150 \mathrm{mg} / \mathrm{dL}$, or current use of antilipidemics with the previously established diagnosis of hyperlipidaemia. The patients were treated with heart failure therapies including cardiac transplantation. After venous blood sample (5-10 mL) collection from each subject, white cell fraction was used to extract DNA according to standard procedure using proteinase $\mathrm{K}$.

\section{VNTR polymorphism genotyping}

Genotyping was performed by polymerase chain reaction (PCR) using the primers previously described ${ }^{15}$. The PCR reaction was carried out in total volume of $25 \mu \mathrm{L}$ containing $150 \mathrm{ng}$ genomic DNA and optimized for the following amplification conditions: $94^{\circ} \mathrm{C}$ for $6 \mathrm{~min}$, then 34 cycles of $94{ }^{\circ} \mathrm{C}$ for $40 \mathrm{~s}, 55^{\circ} \mathrm{C}$ for $30 \mathrm{~s}, 70^{\circ} \mathrm{C}$ for $40 \mathrm{~s}$, and $70{ }^{\circ} \mathrm{C}$ for $8 \mathrm{~min}$. Respective alleles ( $401 \mathrm{bp}$ product -5 repeats allele and $347 \mathrm{bp}$ product - 4 repeats allele) were examined by agarose gel electrophoresis.

\section{Statistics}

The observed number of each genotype was compared with that expected for a population in Hardy-Weinberg equilibrium using the $\chi 2$ test. Fisher's exact test with Tukey-Kramer's method of adjustment for multiple comparisons was used for evaluation of categorical variables. Differences between continuous variables were evaluated using ANOVA with the corresponding post hoc test for more than two groups; multiple regression analysis was applied in all cases of associations between the genotypes and clinical parameters, significant in the univariate analysis. The data analysis was performed using Statistica v. 7.0 (Statsoft Inc., Tulsa, USA) program package. Power calculations were performed using Quanto software ${ }^{23}$.

\section{RESULTS}

A total of 704 unrelated Caucasian subjects ( 372 cases and 332 controls) were enrolled in the study. The basic demographic, clinical and laboratory parameters of the cases are listed in Table 1.

Table 1. Baseline characteristics of the CHF cohorts according to etiology.

\begin{tabular}{lcc}
\hline Variable & $\begin{array}{c}\text { IHD } \\
\text { patients }\end{array}$ & $\begin{array}{c}\text { DCMP } \\
\text { patients }\end{array}$ \\
\hline LVEF (\%) & $25(10-40)$ & $24(10-40)$ \\
NYHA (No. in class & $71 / 130 / 24$ & $57 / 68 / 9$ \\
$\quad$ II/ III/ IV) & & \\
DM I (\%) & 10.6 & 9.6 \\
DM II (\%) & 29.6 & 15.3 \\
HLPP (\%) & 60.4 & 34.0 \\
Anticoagulant agents & 19.5 & 25.6 \\
Digoxin & 54.6 & 83.3 \\
CAA & 5.5 & 2.56 \\
Diuretics & 85.18 & 92.3 \\
BB & 69.4 & 70.5 \\
ACEI & 94.4 & 89.7 \\
AT1 & 3.7 & 10.25 \\
\hline
\end{tabular}

Values are given as median + min-max range

CHF - chronic heart failure, IHD - ischemic heart disease, DCMP - dilated cardiomyopathy, LVEF - left ventricular ejection fraction, NYHA - New York Heart Association Classification, HLPP - hyperlipoproteinemia, CAA - Calcium antagonists, BB - Beta blockers, ACEI - ACE inhibitors, AT1 - Angiotensin type1 receptor blockers 
Associations between examined VNTR in Per3 gene and chronic heart failure

The investigated length polymorphism did not express any deviation from Hardy-Weinberg equilibrium (tested by conventional $\chi 2$ test) in any of the investigated cohorts (the total cases, the cases subdivided according to etiology, the controls). No significant case-control differences were found $\left(P_{\mathrm{g}}=0.302 ; P_{\mathrm{a}}=0.521\right)$. As some sex-linked differences in allele or genotypes of examined polymorphisms were expected, the analysis by gender was performed but no significant sex-dependent associations of genotype distributions or allele frequencies were found. When comparing the genotype and allele frequencies by the most common causes (ischemic heart disease (IHS) vs. dilation cardiomyopathy), no significant differences were found either $\left(P_{\mathrm{g}}=0.585 ; P_{\mathrm{a}}=0.720\right)$.

Multiple regression modeling across the case cohort

In the multiple regression modeling, the VNTR Per3 polymorphism showed no independent predictive role for chronic heart failure risk. In none of the constructed models, was the VNTR Per3 polymorphism associated with increased risk of chronic heart failure or with its etiology (IHD vs. dilated cardiomyopathy). Moreover, the VNTR Per3 polymorphism was not associated with any of the investigated laboratory markers (glycaemia, bilirubin, hematocrit, big endothelin, brain natriuretic factor). In the next step, we tested whether the VNTR Per3 polymorphism had any effect on the ejection fraction or CHF risk. No association of the investigated polymorphism with ejection fraction or NYHA class was found.

\section{DISCUSSION}

Circadian clock regulation has been identified in almost every human cell to date, suggesting a wide-ranging role of a light-dark dependent modulation on physiological and pathophysiological events. According to recent findings of clock genes fluctuating expression within the cardiomyocytes and vascular smooth muscles, there is a strong hypothesis that a clock system may regulate myocardial metabolism ${ }^{24}$. This hypothesis can be further supported by the facts that there is obvious diurnal variation in blood pressure, heart rate and cardiac output. Moreover, it has been known for long time that fatal cardiovascular events, such as acute myocardial infarction, occur preferentially at certain times of the day ${ }^{25}$.

Apropos cardiovascular disorders, we present the first study investigating relationship between polymorphism in Per3 gene and chronic heart failure in a Caucasian population. We found no association of VNTR Per3 polymorphism with chronic heart failure. Further, there was no deviation from the Hardy-Weinberg equilibrium or sex-dependent association of VNTR Per3 genotypes. Our findings are in accordance with recent papers, where certain VNTR alleles were identified with sleep homeostasis rather than with cardiovascular diseases ${ }^{26}$. Recently correlation between VNTR Per3 polymorphism and the extreme diurnal preference (the $5 / 5$ allele associated with morningness and the 4/4 allele with eveningness) has been found ${ }^{18}$. On the other hand, a certain mutation in Per2 gene, which has a similar function in the clock machinery to Per3, has been associated with impaired vascular endothelial function in mice and with familial sleeping disorders in humans ${ }^{27}$. The connection between both could be viewed from the perspective of the possible effect of sleep deprivation on cardiac autonomic control, associating homozygozity in Per3 VNTR with heart rate (HR) and heart rate variability (HRV) $\left(\right.$ ref. $\left.{ }^{20}\right)$. Although no interaction of HR and HRV with VNTR Per3 genotype was found, this polymorphism has been considered a potential marker for individual differences in the autonomic nervous system. It is possible that the Per3 gene more likely modulates the reaction of the heart cell to environmental stimuli and neurohumoral factors and in this way participates in the homeostasis of cardiomyocytes.

Even though the sample consisted of chronic heart patients, we can consider the role of Per3 in circadian neurohumoral regulation and possible disease progression. At a lower level of the circadian cascade, the importance of these regulators in the modulation of cardiovascular functions has been proven in a study on mice with deletions of three clock-controlled PAR bZIP transcription factors ${ }^{28}$. These knockout mice showed increased morbidity, low blood pressure, cardiac hypertrophy and left ventricular dysfunction. Our results suggest that the VNTR Per3 does not influence the severity of the disease but may affect the onset of cardiovascular disorder.

\section{CONCLUSION}

We conclude that Per3 VNTR polymorphism is not associated with chronic heart failure and we do not consider this polymorphism a major risk factor for chronic heart failure or a factor modulating the severity of the CHF in this Caucasian population. Further studies of the relationship of acute and chronic cardiovascular events to the Per3 locus are required.

\section{ACKNOWLEDGEMENTS}

The study was supported by research project IGA NS10206/2009.

\section{CONFLICT OF INTEREST STATEMENT}

The authors stated that there are no conflicts of interest regarding the publication of this article.

\section{REFERENCES}

1. Hastings MH. Central clocking. Trends Neurosci 1997;20:459-64.

2. Dijk DJ, Archer SN. PERIOD3, circadian phenotypes, and sleep homeostasis. Sleep Med Rev 2009;14:151-60. 
3. Lamont EW, James FO, Boivin DB, Cermakian N. From circadian clock gene expression to pathologie. Sleep Med 2007;8:547-56.

4. Shaw E, Tofler GH. Circadian rhythm and cardiovascular disease. Curr Atheroscler Rep 2009;11:289-95.

5. Edery I. Circadian rhythms in a nutshell. Physiol Genomics 2000;3:5974.

6. Hastings MH, Reddy AB, Maywood ES. A clockwork web: circadian timing in brain and periphery, in health and disease. Nat Rev Neurosci 2003;4:649-61.

7. Lowrey PL, Takahashi JS. Mammalian circadian biology: elucidating genome-wide levels of temporal organization. Annu Rev Genomics Hum Genet 2004;5:407-41.

8. Gekakis N, Staknis D, Nguyen HB, Davis FC, Wilsbacher LD, King DP Takahashi JS, Weitz CHJ. Role of the CLOCK protein in the mammalian circadian mechanism. Science 1998;280:1564-9.

9. Sato TK, Yamada RG, Ukai H, Baggs JE, Miraglia LJ, Kobayashi TJ, Welsh DK, Kay SA, Ueda HR, Hogenesch JB. Feedback repression is required for mammalian circadian clock function. Nat Genet 2006:38:312-9.

10. King DP, Takahashi JS. Molecular genetics of circadian rhythms in mammals. Annu Rev Neurosci 2000;23:713-42.

11. Lowrey PL, Shimomura K, Antoch MP, Yamazaki S, Zemenides PD, Ralph MR, Menaker M, Takahashi JS. Positional syntenic cloning and functional characterization of the mammalian circadian mutation tau. Science 2000;288:483-92.

12. Reddy P, Zehring WA, Wheeler DA, Pirrotta V, Hadfield C, Hall JC, Rosbash M. Molecular analysis of the period locus in Drosophila melanogaster and identification of a transcript involved in biological rhythms. Cell 1984;38:701-10.

13. Shearman LP, Jin X, Lee C, Reppert SM, Weaver DR. Targeted disruption of the mPer3 gene: subtle effects on circadian clock function. Mol Cell Biol 2000;20:6269-75.

14. Archer SN, Carpen JD, Gibson M, Lim GH, Johnston JD, Skene DJ, von Schantz M. Polymorphism in the PER3 promoter associates with diurnal preference and delayed sleep phase disorder. Sleep 2010;33:695-01.

15. Benedetti F, Dallaspezia S, Colombo C, Pirovano A, Marino E, Smerald E. A length polymorphism in the circadian clock gene Per3 influences age at onset of bipolar disorder. Neurosci Lett 2008;445:184-7.

16. Ebisawa T, Uchiyama M, Kajimura N, Mishima K, Kamei Y, Katoh M Watanabe T, Sekimoto M, Shibui K, Kim K, Kudo Y, Ozeki Y, Sugishita M, Toyoshima R, Inoue Y, Yamada N, Nagase T, Ozaki N, Ohara O, Ishida N, Okawa M, Takahashi K, Yamauchi T. Association of struc tural polymorphisms in the human period3 gene with delayed sleep phase syndrome. EMBO Rep 2001;2:342-6.
17. Shiromani PJ, Xu M, Winston EM, Shiromani SN, Gerashchenko D, Weaver DR. Sleep rhythmicity and homeostasis in mice with targeted disruption of mPeriod genes. Am J Physiol Regul Integr Comp Physiol 2004;287:47-57.

18. Archer SN, Robilliard DL, Skene DJ, Smits M, Williams A, Arendt J, von Schantz M. A length polymorphism in the circadian clock gene Per3 is linked to delayed sleep phase syndrome and extreme diurnal preference. Sleep 2003;6:413-5.

19. Goel N, Banks S, Mignot E, Dinges DF. PER3 polymorphism predicts cumulative sleep homeostatic but not neurobehavioral changes to chronic partial sleep deprivation. PLoS One 2009;4(6):e5874.

20. Viola AU, James LM, Archer SN, Dijk DJ. PER3 polymorphism and cardiac autonomic control: effects of sleep debt and circadian phase. Am J Physiol Heart Cirk Physiol 2008; 295:2156-63.

21. Bray MS, Shaw CA, Moore MW, Garcia RA, Zanquetta MM, Durgan DJ, Jeong WJ, Tsai JY, Bugger H, Zhang D, Rohrwasser A, Rennison JH, Dyck J, Litwin SE, Hardin PE, Chow CHW, Chandler MP, Abel ED, Young ME. Disruption of the circadian clock within the cardiomyocyte influences myocardial contractile function, metabolism, and gene expression. Am J Physiol Heart Cirk Physiol 2008;294:1036-47.

22. Sheward WJ, Naylor E, Knowles-Barley S, Armstrong JD, Brooker GA Seckl JR., Turek FW, Holmes MC, Zee PC, Harmar AJ. Circadian control of mouse heart rate and blood pressure by the suprachiasmatic nuclei: behavioral effects are more significant than direct outputs. PLoS One 2010;5(3):e9783.

23. Gauderman W.J. Sample size requirements for association studies of gene-gene interaction. Am J Epidemiol 2002;155:478-84.

24. Young ME, Razeghi P, Taegtmeyer H. Clock genes in the heart: characterization and attenuation with hypertrophy. Cirk Res 2010;88:114250 .

25. Young ME. The circadian clock within the heart: potential influence on myocardial gene expression, metabolism, and function. Am J Physiol Heart Cirk Physiol 2006;290:1-16.

26. Nadkarni NA, Weale ME, von Schantz M, Thomas MG. Evolution of a length polymorphism in the human PER3 gene, a component of the circadian system. J Biol Rhythms 2005;20:490-9.

27. Viswambharan H, Carvas JM, Antic V, Marecic A, Jud C, Zaugg CE, Ming XF, Montani JP, Albrecht U, Yang Z. Mutation of the circadian clock gene Per2 alters vascular endothelial function. Circulation 2007;115:2188-95.

28. Wang Q, Maillard M, Schibler U, Burnier M, Gachon F. Cardiac hypertrophy, low blood pressure, and low aldosterone levels in mice devoid of the three circadian PAR bZip transcription factors DBP, HLF, and TEF. Am J Physiol Regul Integr Comp Physiol 2010;299(4):1013-9. 